\title{
Narrativas históricas de los jóvenes: Una cara de su orientación temporal
}

Doi: https://doi.org/ 10.25100/hye.v15i53.8741

Artículo recibido: 12-08-2018 Artículo aceptado: 14-05-2019

\section{Maria Isabel Gomes Barca Oliveira}

Licenciada en Historia, además cuenta con un Máster en Educación de Ciencias sociales y un Doctorado en Historia de la Educación. Hace parte del Grupo de Investigación Educación y Desafíos Sociales y es profesora de la Facultad de Letras de La Universidad de Porto.

Facultad de Letras da Universidade do Porto (FLUP), Portugal.

Correo electrónico: isabarca@clix.pt

ORCID: 0000-0001-7921-6928

Forma de citar este artículo: Gomes Barca Oliveira, Maria Isabel. "Narrativas históricas de los jóvenes: Una cara de su orientación temporal”. Historia y Espacio, vol. 15 n 53 (2019): 309-332. doi. org/ 10.25100/hye.v15i53.8741.

Artículo Tipo 2: de reflexión. 


\section{Narrativas históricas de los jóvenes: Una cara de su orientación temporal}

Resumen: La narrativa histórica permite acceder al modo como un autor concibe el pasado y como utiliza los elementos estructurales del saber histórico, por ejemplo, la interpretación de fuentes, descripción y explicaciones sobre lo que pasó y otros rasgos de la conciencia histórica en tanto que componentes para una orientación temporal. Por todo ello, la narrativa debe ser también cultivada por quien aprende Historia. En este artículo se discuten dos supuestos -la narrativa como un aspecto de la conciencia histórica y el aprendizaje histórico como construcción del pensamiento histórico- que se consideran fundamentales para la educación histórica actual. Para un diálogo conceptual entre estas propuestas se presentan, a) algunos conceptos estructurales que componen la narrativa histórica como elementos clarificadores de lo que significa desarrollar el pensamiento histórico de los alumnos y, b) algunos resultados de estudios sobre las ideas de los jóvenes en Historia, de naturaleza predominantemente cualitativa. Se discuten, específicamente, narrativas 'históricas' de jóvenes que permiten interpretar sus ideas en el plano substantivo y estructural, incluyendo sentimientos de identidad colectiva. Por último, se formulan consideraciones sobre las relaciones entre la investigación en educación histórica y las posibilidades de desarrollar el pensamiento histórico de los alumnos en las clases de Historia.

Palabras clave: Pensamiento histórico, Narrativas y aprendizaje en Historia, Conciencia histórica de los jóvenes.

\section{Historical narratives of young people: A face of their temporal orientation}

Abstract: Historical narrative allows us to access the way the author conceives the past and how she uses the structural elements of historical knowledge, for example, source interpretation, description, explanations of what happened, and various other traits of historical consciousness as ingredients for temporal orientation. Due to all these facets, the narrative must also be cultivated by those who learn History. In this article we discuss two presuppositions - narrative as a face of historical consciousness and historical learning as construction of historical thought - that are considered fundamental for current history education. For a conceptual dialogue between such presuppositions we present, a) some structural concepts that compose the historical narrative as clarifying elements of what it means to develop students historical thought and, b) some results of predominantly qualitative studies on ideas of young people in History. Particular discussion is made of 'historical' narratives of young people to allow the understanding of their ideas at the substantive and structural levels, including the meanings of collective identity. Finally, we consider the relations between research in history education and the possibilities of developing the historical thinking of students in history class.

Keywords: Historical thought, Narratives and learning in History, Historical consciousness of young people. 


\section{Introducción}

Conocer el pasado - ¿De dónde vengo?-constituye una necesidad intrínseca del ser humano: nos lleva, por lo menos, a buscar nuestras raíces más próximas. Pero el ser humano está (o tiende a estar) siempre ávido de conocer más y mejor este mundo - ¿Dónde estoy?- en el sentido de poder actuar de forma racional y, así, intentar alejarse de las consecuencias nefastas de sus acciones y conseguir algo que considere positivo según su perspectiva. Por lo tanto, aprender a pensar históricamente es esencial en la formación escolar de los niños y de los jóvenes para contribuir a la ampliación objetiva de sus horizontes en el mundo en que viven. Y es a través de la narrativa histórica, en sus diversas formas y lenguajes, que el pensamiento histórico y la conciencia histórica se aclaran y expresan. Estos son algunos de los ejes fundamentales que constituyen la base epistemológica de la investigación en Educación histórica.

\section{La idea de conciencia histórica}

La Historia como disciplina científica hace parte del impulso de cada uno por buscar situarse y actuar de forma más adecuada por medio de la comprensión de la vida de otros, en diversos tiempos y lugares. Es en esta línea que muchos pensadores ${ }^{1}$ han debatido la relevancia de la Historia para el ser humano teniendo en cuenta, de forma tácita o explicita, su orientación temporal. La matriz de la conciencia histórica presentada y discutida por Jörn Rüsen ${ }^{2}$ identifica filosóficamente y de forma fructífera la idea de los usos genuinos de la Historia en la búsqueda de crear mejores horizontes de expectativa en cada sujeto. Esta concepción rechaza epistemológicamente argumentos simplistas sobre la función de la Historia, como la que sostiene que este conocimiento sirve apenas para un diálogo sobre curiosidades en discusiones entre elites formadas, o hasta para ser usada como "maestra de vida" sin atender las variables propias de la historia humana, siempre imbuida de cambios y continuidades. Sin embargo, a pesar de este reparo, se asume aquí que la comprensión de la Historia también vehiculiza emoción y algunas lecciones para la vida. La

1 Como ejemplos relevantes ver: Raymond Martin, The past within us (Princeton: Princeton University Press, 1980); Peter Lee, "Por qué aprender História?'[versão portuguesa de 'Why Learn History', 1984]. Educar em Revista, No. 42 (2011): 19-42; Jörn Rüsen, A Razão da História. Teoria da História: os fundamentos da ciência histórica (Brasília: UniB, 2001).

2 Ver, entre varias de las obras de Jörn Rüsen, su artículo de inicios de la década de 1990. Jörn Rüsen, "The development of narrative competence in historical learning: an ontogenetic hypothesis concerning moral consciousness", en Studies in metahistory, ed. Peter Duvenage (Pretoria: Human Sciences Research Council, 1993), 63-84. 
Historia aunque no ofrece predicciones fijas para el presente y mucho menos para el futuro, no deja de ser indispensable para una orientación temporal mas avanzada, sobretodo en las sociedades actuales en las que los cambios vertiginosos impiden a las personas continuar con sus rutinas y expectativas tradicionales. Esta condición de rupturas continuas no evita que se encuentren semejanzas y continuidades entre el pasado y el presente; el ejercicio mental sobre el peso relativo que esas semejanzas y diferencias representan para evaluar escenarios posibles de futuro es una llave potencial de una orientación temporal avanzada. Es necesario tratar de entender por qué sucede lo que sucede. Es necesario tratar de distinguir entre la información genuina (siempre a partir de un punto de vista dado) y la propaganda (dirigida a sesgar los hechos). En esta visión epistemológica, el sujeto asume un papel relevante: es ella o el quienes van a buscar en la Historia alguna respuesta para orientarse mejor, es ella o el quienes producirán respuestas más o menos validas - algunas al nivel historiográfico-para proporcionar una orientación temporal, es ella o el quienes acogerán esas respuestas historiográficas para situarse y actuar en su tiempo.

Todo esto justifica, y hasta exige, la integración de la disciplina de la Historia en los currículos escolares para todos los niños y jóvenes. Desde temprana edad, el ser humano busca situarse dentro de la familia y el entorno que lo rodea. Mediante la Historia, a partir de ese medio más próximo ellas y ellos pueden entender mejor como funciona este mundo que, incluso para los más jóvenes, se vuelve algo familiar a través de los medios audiovisuales con los cuales tienen contacto diariamente. La comprensión progresiva de la vida de otras personas que la Historia les proporciona, personas con diversas formas de vida semejantes o hasta aparentemente muy extrañas, les da la oportunidad de ampliar sus horizontes de presente y de futuro. También les da la posibilidad de reconocerse mejor como seres en comunicación con otros seres, y lo importante que esto es para los agentes corresponsables de lo que sucede y lo que vendrá.

Reflexiones filosóficas recientes de Rüsen sobre la interpretación en Historia ${ }^{3}$ agregan a este debate una visón que integra una actitud constructiva en el análisis crítico del relativismo pos moderno; al mismo tiempo, acoge con beneplácito el papel interpretativo del sujeto en la Historia, pero crítica el subjetivismo exagerado; está de acuerdo con la necesidad de revisar diferentes perspectivas, pero defiende criterios de validación de las diferentes posiciones;

3 Jörn Rüsen, "Humanismo Intercultural: ideia e realidade”, en Humanismo e Didática da História: Jörn Rüsen, eds. Maria Auxiliadora Schmidt et al. (Curitiba: W. A. Editores, 2015), 133-152. 
reconoce algunos fundamentos para la deconstrucción que la corriente relativista cultiva, pero sin dejar de reforzar una posición de objetividad crítica, dialógica que puede designarse "intersubjetividad".

En la aplicación de esta idea de intersubjetividad de nivel académico al ambiente del cotidiano de cada sujeto se ajusta la propuesta de un humanismo intercultural. Por un lado, se percibe que cada sujeto puede acoger de forma no conflictiva diversas dimensiones de identidad colectiva familiar, local, nacional, profesional, cultural y global. Por otro lado, se enfatiza la formación de la conciencia de una humanidad compartida dentro de la diversidad-para lo cual el ejercicio de empatía histórica con relación al "otro" puede contribuir-e invita a reforzar el diálogo sereno y, por lo tanto, a la reducción de odios entre culturas.

La narrativa histórica, en un sentido amplio de la expresión de ideas sea de forma escrita, por el diálogo, por medio de la imagen, tiende a indicar esta conceptualización que el sujeto construye acerca del pasado en su relación con el presente y, eventualmente, con perspectivas conscientes de futuro. Por tal razón, la narrativa histórica refleja la conciencia histórica de quién la produce y de quién se adhiere a ella.

Estas reflexiones de carácter humanista son marcadores importantes para la construcción de un pensamiento histórico genuino y que ha llevado a investigar “¿cómo piensan los niños y los jóvenes en Historia?”.

\section{Epistemología de la Historia e investigación en educación histórica.}

\section{Estudios Iniciales}

En la década de 1970, emerge en Inglaterra una línea de investigación con el fin de encontrar respuestas sustentadas a la cuestión de las posibilidades de educación histórica de niños y jóvenes. Este movimiento tiene el propósito de combatir una tendencia curricular de la época, de menosprecio de la disciplina de Historia como efecto de una aplicación mecanicista de la teoría de los estadios de desarrollo cognitivo (por grupos de edad) al aprendizaje histórico. En el debate por la Historia en el currículo escolar participan historiadores, filósofos de la Historia, investigadores en Educación. Algunos de ellos ejecutan proyectos de investigación en el terreno de las escuelas para indagar, con base en presupuestos epistemológicos de la Historia (y no en presupuestos generales tendencialmente inspirados en las ciencias naturales), cómo es que los niños y jóvenes dan sentido a la Historia. Entre estos estudios, dos 
se destacan como pilares fundamentales para la investigación internacional en educación histórica ${ }^{4}$ : el estudio exploratorio de carácter esencialmente cualitativo, desarrollado por Alaric Dickinson y Peter Lee ${ }^{5}$ con alumnos entre los 12 y 18 años sobre explicación histórica, y el proyecto sobre enseñanza y aprendizaje de la Historia en situación de clase, un estudio longitudinal de características de investigación experimental desarrollado durante tres años con alumnos de Historia entre los 13 y los 16 años, y coordinado en la última fase por Dennis Shemilt ${ }^{6}$. En los dos casos, las propuestas de trabajo presentaban temas históricos y fuentes de soporte para permitir a los estudiantes pensar históricamente: a) sobre la explicación, es decir, los "porqués" de las decisiones tomadas por los agentes históricos en un contexto dado, y b)sobre evidencias, o sea, las bases empíricas que permiten conocer el pasado en Historia. Es importante aclarar los sentidos epistemológicos atribuidos a estos dos conceptos en los estudios referidos. En primer lugar, explicación y evidencia son conceptos estructurales del saber histórico; están más allá de los conceptos substantivos (por ejemplo revolución, democracia, industrialización o ilustración) y, por eso, son también denominados conceptos de segundo orden. Entonces es necesario aclarar en qué sentido se utilizan dichos conceptos.

a. La explicación histórica es aquí entendida como explicación intencional, en línea con el pensamiento de Collingwood ${ }^{7}$, esto es, enfocada en la comprensión de las acciones de los sujetos en el pasado, y teniendo en cuenta su ambiente cultural. En esta acepción, el historiador usa su imaginación informada para "recrear" mentalmente lo que los seres humanos decidieron e hicieron en una determinada situación pasada y, con ese fin, el autor busca explicaciones a la luz de motivos, disposiciones, intenciones y objetivos de agentes históricos así como consecuencias esperadas o no. Con relación a este concepto epistemológico de explicación intencional, las ideas de los alumnos participantes en

4 Una descripción breve de estos dos proyectos puede leerse también en la obra reciente de Maria Auxiliadora Schmidt et al.. Aprendizagem Histórica: Catálogo Seletivo de Teses e Dissertações Brasileirase Portuguesas (Curitiba: W.A. Editores, 2018); y en: Isabel Barca, O Pensamento histórico dos jovens (Braga: CEEP, Universidade do Minho, 2000).

5 Alaric Dickinson y Peter Lee, History teaching and historical understanding (London: Heineman, 1978).

6 Denis Shemilt, History 13-16: Evaluation Study (Edinburgh: Holmes McDougall, 1980).

7 Robin George Collingwood, The Idea of History (Oxford: Clarendon Press, 1946). 
las investigaciones iniciales mostraron una diversidad de niveles en los cuales el pensamiento histórico mas elaborado no se verificaba necesariamente solo entre alumnos entre los 16 y 18 años; algunos alumnos de este grupo de edad mostraron ideas muy inconsistentes, lo que contrasta con cierta sofisticación de ideas de algunos alumnos de menor edad. Estos y otros resultados posteriores sugieren que, en cada sujeto, los niveles conceptuales - sobre todo los más incipientespueden sufrir oscilaciones, esto es, los niveles de respuesta de cada sujeto pueden retroceder o avanzar a lo largo de diferentes tareas que les sean propuestas. Estos estudios tuvieron una alta importancia para refutar el determinismo de los estadios de desarrollo cognitivo por grupos de edad, el cual había llevado a una conclusión generalizante de que sólo a los 16 años sería posible que los alumnos fuesen capaces de entender la Historia.

Tal vez para evitar algunas ideas falsas y críticas inconsecuentes del principio "collingwoodiano" de imaginación histórica en torno a la recreación de un pasado humano en estudio - que nada tiene que ver con la fantasía, pero si con una sofisticada labor mental apoyada en aquello que la evidencia obliga a aceptar- algunos autores pasaron a utilizar el termino "empatía histórica", expresión que, sin embrago, también suscita ambigüedades por la posible confusión con la simpatía, que es un concepto principalmente del ámbito emocional. Bajo el enfoque de la explicación intencional pero ya desde la designación de empatía histórica, Denis Shemilt ${ }^{8}$, y Ashby y Lee ${ }^{9}$ presentaron posteriores modelos de categorización de ideas de los alumnos que avanzan en convergencia. Los niveles de pensamiento histórico sugeridos por el análisis de las ideas de los alumnos participantes pasan por nociones más ingenuas de que la historia humana es simplemente algo extraño (pereciendo incomprensible, confusa o inútil porque trata de gente más atrasada); otras que establecen un puente, aunque frágil, entre el presente y el pasado en el cual las acciones de los agentes históricos son afrontadas

8 Denis Shemilt, "Beauty and the philosopher: Empathy in history and the classroom", en Learning history, eds. Alaric Dickinson, Peter Lee y Peter Rogers (London: Heinemann, 1984), 39-84.

9 Rosalyn Ashby y Peter Lee, "Children's concepts of empathy and understanding in history", en The History Curriculum for Teachers, ed. Christopher Portal (London: The Falmer Press, 1987), 62-88. 
con seriedad pero sólo de acuerdo con los principios de experiencias presentes (estereotipos y "presentismo"), y; otras ya más elaboradas que indican reconocer semejanzas y diferencias entre situaciones del pasado $\mathrm{y}$ del presente, de forma emergente $\mathrm{o}$ atendiendo al contexto propio de cada época y espacio.

b. En lo concerniente a la evidencia en Historia, Shemilt ${ }^{10}$ y Ashby ${ }^{11}$ establecieron niveles progresivos de elaboración conceptual de los alumnos en cuanto a la interpretación de fuentes para la comprensión del pasado: 1) desde ideas más ingenuas en las cuales la validez empírica de las fuentes históricas (¿Qué aconteció realmente?) no es considerada, 2) pasando por niveles intermedios en los que la interpretación se basa en principios un poco más elaborados que pueden ser: 2.1 el reconocimiento de que la información sobre el pasado es conocida por una autoridad incuestionable en la materia (historiadores, profesores, libros de Historia); 2.2. la comprensión de que existen "testimonios" con diversos grados de validez siendo más valorizados los testimonios directos de los acontecimientos; 2.3. desde una visión de "cortar y pegar" la cual presupone que las conclusiones históricas se construyen utilizando diversas fuentes primarias y secundarias, y 3) en modos más avanzados, cuando algunos alumnos muestran que alcanzan nociones más elaboradas de evidencia histórica al establecer inferencias; 3.1 . ya sea a partir de marcos restringido; 3.2. o incluso en un contexto específico, con base en fuentes de perspectivas variadas (lo que hace que estos alumnos busquen la mejor respuesta para comprender un determinado asunto por la interpretación de fuentes diversas que tiene en mente).

\section{Desarrollos de investigación en educación histórica}

Conceptos tales como explicación (intencional y causal), evidencia, múltiples perspectivas, objetividad, cambio, significancia ${ }^{12}$ son esenciales a la naturaleza

${ }^{10}$ Denis Shemilt, "Adolescent ideas about evidence and methodology in history", en The History Curriculum for Teachers, ed. Christopher Portal (London: The Falmer Press, 1987), 39-61.

${ }^{11}$ Rosalyn Ashby, "O conceito de evidência histórica: exigências curriculares e concepções dos alunos”, en Educação Histórica e Museus. Actas das II Jornadas Internacionais de Educação Histórica, coord. Isabel Barca (Braga: CIEd, Universidade do Minho, 2003), 37-57.

${ }^{12}$ El termino "Relevancia” proviene de la traducción de significance, un termino utilizado en la investigación en educación histórica. Podría traducirse a la lengua portuguesa como relevancia; sin embrago, se mantiene aquí el termino "significancia" bajo el argumento de 
del saber histórico. Por consiguiente, teniendo en cuenta su clarificación a la luz de varias corrientes epistemológicas, tales conceptos pueden y deben ser objeto particular no sólo del debate filosófico/metodológico, sino también del examen en el plano de las ideas de los sujetos incluyendo las de niños y jóvenes.

Este marco teórico brinda pistas muy desafiantes para el análisis del pensamiento histórico de los estudiantes - creemos que en cualquier parte del mundo- y vuelve posible avanzar en la conciencia del papel formativo de la educación histórica sin caer en sincretismos nebulosos o en positivismos basados en una verdad fija. En el campo de la educación histórica, al analizar las manifestaciones de pensamiento histórico que niños y jóvenes van construyendo, incluso a través de sus relatos narrativos, es posible identificar no sólo que retratos substantivos construyen sino a la vez que conceptos de segundo orden ellos tácitamente desarrollan. Es posible también observar la orientación historiográfica que indican. Por ejemplo: cronologías, comentarios generales o meros relatos sobre lo que pasó, sean fragmentados o no, apuntan a un pensamiento descriptivo; relatos que contemplan explicaciones sobre lo que pasó sugieren un pensamiento del tipo de "gran narrativa" o de multiperspectiva, sea en una postura relativista de que todo vale o en un marco de intersubjetividad discutida y algo equilibrada. Cabe aquí resaltar que un relativismo total (¿absoluto?) ha sido perjudicial en la Educación de los jóvenes. Existen varios puntos de vista que deben ser escuchados y debatidos (por el principio de intersubjetividad) pero algunos pueden ser considerados más poderosos que otros en el campo conceptual o moral.

Por consiguiente, en esta línea, y dado que en la educación sólo se puede cambiar conscientemente aquello que se conoce, para explorar la progresión conceptual de los jóvenes en Historia se requiere de una perspectiva investigativa que promueva la clarificación de las ideas substantivas y de segundo orden (estructurales) que los alumnos construyen, así como los usos que indican, por ejemplo, al nivel de la construcción de sus identidades sociales. Estos pilares de investigación en educación histórica han influenciado los enfoques de estudio sobre enseñanza y aprendizaje de la Historia en diversos países y, dentro de tales presupuestos, uno de los caminos ha sido el de encontrar modelos de ideas de alumnos en un progresión que tenga en cuenta la naturaleza de la Historia. Esa progresión no debe basarse únicamente en auscultar conocimientos adquiridos en cuantas descripciones y explicaciones que les fueron transmitidas como

que en la lengua portuguesa éste ya es utilizado en estadística (este último uso se da de igual forma en castellano). 
si fuesen verdades; ella debe incluir un "pensar históricamente" que implica saber interpretar fuentes, sacar conclusiones y debatir diversas perspectivas.

La variabilidad de las perspectivas existentes se relaciona con la forma en la que cada uno, incluyendo los alumnos, selecciona y atribuye significancia al pasado. Entre los varios estudios sobre la significancia que los alumnos dan a situaciones, hechos y personajes históricos se destacan aquí los de Seixas ${ }^{13}$, por su carácter pionero ya que abrió nuevas posibilidades de dar sentido a los sentidos que los jóvenes manifiestan acerca de la Historia, y el de Cercadillo ${ }^{14}$ por loa avances en la conceptualización sobre significancia histórica que presenta. Seixas construye una tipología de ideas de alumnos sobre significancia histórica - posturas subjetivistas, objetivista, narrativista- que han influenciado muchos estudios a nivel internacional. Cercadillo generó un modelo de progresión conceptual de ideas de alumnos en los que variados tipos de significancia - contemporánea, causal, estándar, simbólica y de inter-relación con el presente y el futuro- son combinados con otros indicadores ligados también a criterios históricos, a saber, la consideración de la significancia como fija o variable. Entre los participantes en esta investigación, los alumnos más jóvenes tienden a situarse en una atribución de significancia causal y estándar, fija o variable, y los alumnos mayores presentan en mayor proporción niveles de significancia mas elaborados (variables, dentro de tipos más allá de lo causal). Este modelo ha inspirado varios estudios en Portugal sobre tipos de significancia que alumnos y profesores atribuyen a varias situaciones históricas, los cuales también sugieren una incidencia en la atribución de significancia de tipo causal, sea fija o variable ${ }^{15}$. Las investigaciones centradas en el análisis de la significancia histórica de personajes o de hechos históricos atribuida por alumnos de $9^{\circ}$ año de escolaridad, se basó en la tipología de ideas de alumnos sobre significancia histórica creada por Peter Seixas ${ }^{16}$.

${ }^{13}$ Peter Seixas, "Mapping the terrain of Historical Significance”. Theory and Research in Social Education, 61,1 (1997): 22-27.

${ }^{14}$ Lis Cercadillo, "Significance in history: students' ideas in England and Spain”, en Raising Standardsin History Education. International Review of History Education, eds. Alaric Dickinson, Peter Gordon y Peter Lee (London: Woburn Press, 2001), 116-145.

${ }^{15}$ Ver Júlia Castro, “A Interculturalidade e o Pensamento Histórico dos Jovens” (Tesis de doctorado, Universidade do Minho, 2006), y Olinda Alves, "Concepções de professores e de alunos sobre significância histórica - um estudo no $3^{\mathrm{o}}$ ciclo do ensino básico" (Tesis de Maestría, Universidade do Minho, 2007)

${ }^{16}$ La tipología de análisis presentada por Peter Seixas (op cit.), inspiró varios estudios en Portugal. Como ejemplos ver Márcia Monsanto, "Concepções de alunos sobre Significância 
Otro concepto intrínsecamente ligado a los presupuestos de cada escuela historiográfica es el de la comprensión del cambio en Historia. Keith Barton $^{17}$, al analizar datos sobre los sentidos de cambio histórico atribuidos por alumnos, en los Estados Unidos y en Irlanda del Norte, encontró tres tendencias diferentes: Los alumnos norte-americanos conciben el cambio como evolución, en el sentido de progreso lineal y como resultado de la acción individual; los alumnos irlandeses incorporan la idea de diversidad de situaciones, tanto en el sentido de progreso como simplemente de cambio en función de factores sociales. Es de anotar que, aunque el autor no asume un modelo de progresión conceptual como presupuesto de su análisis de datos, presenta una diferenciación entre dos patrones de ideas sobre cambio histórico, en la cual uno es tácitamente considerado más elaborado (perspectivado) en comparación al otro. En Portugal, un estudio ${ }^{18}$ inspirado en lo que Keith Barton planteó, sugirió que las ideas de los participantes portugueses se aproximan más a las de sus colegas irlandeses: reconocen la coexistencia de situaciones aparentemente contrastantes, conciben cambios en el progreso pero rara vez de forma lineal, señalando factores sociales (señalan aspectos positivos y negativos en simultaneo, a veces en función de diferentes grupos sociales).

El presupuesto de que un enfoque histórico avanzado considera diversos puntos de vista ha llevado a muchos investigadores a integrar el elemento de múltiples perspectivas en sus estudios sobre ideas históricas de alumnos. Entre ellos, es de destacar el trabajo en Irlanda del Norte, en el que investigadores fomentaron al interior de los salones de clase la interpretación de perspectivas históricas de los "dos lados" del conflicto en esta región y que hoy hace parte del currículo de Historia en Irlanda del Norte ${ }^{19}$. Este enfoque parece haber

Histórica no contexto da História de Portugal: um estudo com alunos do $3^{\mathbf{0}}$ ciclo do Ensino Básico" (Tesis de Maestría, Universidade do Minho, 2004); Fátima Chaves, "A significância de personagens históricas na perspectiva de alunos portugueses e brasileiros" (Tesis de Maestría, Universidade do Minho, 2006).

${ }^{17}$ Keith Barton, "Ideias das crianças acerca da mudança através dos tempos: resultados de investigação nos Estados Unidos e na Irlanda do Norte”, en Perspectivas em Educação Histórica. Actas das I Jornadas Internacionais de Educação Histórica", coord. Isabel Barca (Braga: CEEP, Universidade do Minho, 2001), 55-68.

${ }^{18}$ Ver Elvira Machado, “Mudança em História - concepções de alunos do 7. ano de escolaridade” (Tesis de Maestría, Universidade do Minho, 2006).

${ }^{19}$ Ver Keith Barton and Alan McCully, "When History teaching really matters - Understanding the impact of school intervention on students' neighbourhood learning in Northern Ireland". International Journal of Historical Learning Teaching and Research, 8, 1 (2009): 28-46; Alan 
contribuido a una visón histórica más equilibrada (y quizás más dialogante) entre jóvenes de comunidades diferentes. También en Portugal y en Brasil el asunto de las múltiples perspectivas en Historia ha suscitado especial interés, con varios estudios desarrollados sobre las ideas de alumnos desde este enfoque investigativo ${ }^{20}$. Nótese que explorar diferentes perspectivas en Historia no es tarea fácil. Para colocar a los alumnos a interpretar un conjunto de fuentes diversas sobre un dato pasado ellos necesitan de, progresivamente, saber que las afirmaciones históricas son inferencias basadas en las fuentes disponibles y no simplemente copias de la información contenida en ellas; que las explicaciones del pasado no son meras afirmaciones factuales y que la existencia de diferentes versiones históricas no significa que cada una sea una mera cuestión de opinión.

Además de los ejemplos anteriores, es de destacar que, paulatinamente en Portugal, varios profesionales ligados a la investigación en Enseñanza de la Historia han comenzado a explorar tanto las concepciones de profesores y las que son transmitidas por los libros de texto, como los procesos de aprendizaje y las prácticas de enseñanza desde la perspectiva -nótese- de la inclusión de ideas estructurales de la Historia ${ }^{21}$.

McCully, "A question of identity? Purpose, policy and practice in the teaching of history in Northern Ireland and the Republic of Ireland". International Journal of Historical Learning Teaching and Research, 11, 2 (2013): 145-158.

${ }^{20}$ El presupuesto de la pluralidad de perspectivas en Historia está patente, por ejemplo en los trabajos de Marília Gago, "Children's understanding of historical narrative in Portugal", en Understanding History: Recent research in in history education, eds. Rosalyn Ashby, Peter Gordon y Peter Lee (London: Routledge Falmer 2005), 83-97; Manuela Carvalho y Isabel Barca, “Gli studenti e le fonti in tre paesi europei”. I Quadreni di Clio'92, 13 (2014): 95-114; Marcelo Fronza, "Os jovens e as ideias de objetividade e verdade na aprendizagem histórica sobre o passado a partir das histórias em quadrinhos”, en Educação e Consciência Histórica na Era da Globalização, coord. Isabel Barca (Braga: CIED, Universidade do Minho/Associação de Professores de História, 2011), 179-200; Eder de Souza "Cinema e Educação histórica: Jovens e a sua relação com a História nos filmes” (Tesis de doctorado, Universidade Federal do Paraná, 2014).

${ }^{21}$ Ver el estudio reciente de Mariana Lagarto "Desenvolver e avaliar competências em História. Um estudo com professores do $3^{\underline{0}}$ ciclo do ensino básico” (Tesis de doctorado, Universidade do Minho, 2017). Se debe tener en cuenta que el currículo de Historia de enseñanza básica y los exámenes de Historia al final de la enseñanza secundaria ya reflejan algunas de estas propuestas de educación histórica. Ver Paulo Abrantes, Currículo nacional do Ensino Básico - Competências essenciais (Lisboa: DEB, Ministério da Educação, 2001); Helena Veríssimo, "A avaliação de competências históricas através da interpretação da evidência" (Tesis de doctorado, Universidade do Minho, 2013). 


\section{Narrativas históricas, narrativas de jóvenes}

Los conceptos estructurales resaltados aquí, y otros, se encuentran mezclados en la producción historiográfica y, algunos de ellos, pueden ser sugeridos por la narrativa histórica que los sujetos presentan en diversos niveles de elaboración. Sin embargo, se habla mucho en la narrativa, pero en Historia esta noción es bien compleja, ya que su sentido puede variar conforme las escuelas historiográficas en las que trabajan los historiadores. La narrativa histórica puede transmitir la idea de una oposición entre la Historia centrada en la descripción versus la Historia explicativa y estructural, y puede entenderse como la forma habitual de producción historiográfica que abarca cualquier escuela, sean las marxistas con una visón dialéctica por estadios sobre el "proceso histórico", la "rankeana" de tipo descriptivo, la estructuralista de los Annnales, las post-estructurales como las relativistas y estructuralistas ${ }^{22}$, las "perspectivistas" de influencia collingwoodiana. Ante esta diversidad de modelos, Atkinson, Dray o Fulbrook ${ }^{23}$, entre varios que defienden la naturaleza descriptiva/explicativa y perspectivada de la producción historiográfica, argumentan que la diversidad de perspectivas es una característica de la naturaleza de la Historia dada la propia diversidad de su objeto: acontecimientos y situaciones del pasado humano pueden ser seleccionados, interpretados y evaluados sobre diversos puntos de vista, en diversas escalas y a partir de diversos ángulos. Esto no obliga a que se niegue un puente conceptual para acceder a una cierta realidad en el pasado; ese puente existe por los indicios materiales e inmateriales que dejo. Los hechos existieron, y su relación es la que puede variar. Este elemento selectivo de la investigación histórica se interrelaciona con el tipo de significancia que cada uno atribuye al pasado. En una perspectiva tradicional será una relevancia fija y ligada intrínsecamente a ciertos hechos en si (como si "hablasen por si mismos"); en una perspectiva más actualizada será variable según en función de las formas como cada historiador selecciona acontecimientos, personajes y situaciones. Ahora, la producción historiográfica actual no se limita a una selección factual. La relevancia o significancia que orienta las interpretaciones y explicaciones tiende a introducir aún más variabilidad en las conclusiones históricas, lo que no significa que se tenga que caer en un relativismo total. Su relativa validez y

\footnotetext{
${ }^{22}$ Christopher Lloyd, The structures of history (Oxford: Basil Blackwell,1993).

${ }^{23}$ R. Atkinson, Knowledge and explanation in history: An introduction to the philosophy of history (London: Macmillan, 1978); William Dray, Perspectives on history (London: Routledge and Kegan Paul, 1980); Mary Fulbrook, Historical theory (London: Routledge, 2002).
} 
consistencia lógica debe ser debatida y evaluada, especialmente dentro de la comunidad académica.

El concepto de narrativa puede tener un significado aún más amplio, extensiva a la comunicación en cualquier campo de conocimiento; puede significar la expresión de una cierta perspectiva que el comunicante quiere presentar y que puede ser transmitida por varios lenguajes, sean orales o escritos, audiovisuales, fílmicas, u otras. En Historia (y no sólo), el poder simbólico de la narrativa en cuanto forma de comunicación abre la puerta para la comprensión de las formas de identidad colectiva que los jóvenes interiorizan. Por todas estas razones, como señaló Rüsen ${ }^{24}$ la narrativa puede ser considerada una cara material de la conciencia histórica. Por la narrativa histórica se gana acceso al modo como su autor concibe el pasado y utiliza sus fuentes, así como los tipos de significancia y sentidos de cambio atribuidos a la Historia. También refleja, tacita o explícitamente, un cierto tipo de conciencia histórica, esto es, las relaciones que su autor encuentra entre el pasado, el presente y, eventualmente, el futuro, en el campo social y también personal.

Existen, por lo tanto, varias perspectivas sobre lo que es la narrativa en Historia. La narrativa histórica que aquí se defiende es una narrativa construida con base en la evidencia disponible, que considera varios puntos de vista y, por lo tanto, se aleja del retrato homogéneo del pasado que constituye la "gran narrativa", tal como es referida frecuentemente en los estudios de educación histórica y en debates filosóficos. Las versiones pueden ser más o menos validas en lo que respecta a su adecuación a criterios históricos para una buena -siempre provisoria- respuesta a una pregunta dada sobre el pasado. Pero también se acepta que varias versiones puedan competir entre si y mantenerse en la medida que no fueran refutadas con base en criterios metodológicos propios de la Historia. Este es un debate desde el punto de vista del realismo crítico el cual está relacionado con la problemática de la objetividad de las "conclusiones" históricas y de su naturaleza provisoria.

Dentro de esta última línea epistemológica, en Portugal la investigación vinculada a la educación histórica avanzó hacia un conjunto de estudios centrados en la preocupación de explorar la conciencia histórica de los

\footnotetext{
${ }^{24}$ Ver, entre varias obras de Rüsen, su artículo de inicios de la década de 1990, "The development of narrative competence in historical learning", 63-84, y su libro traducido a lengua portuguesa, A Razão da História: os fundamentos da ciência histórica (Brasília: UniB, 2001).
} 
jóvenes ${ }^{25}$. En este ámbito, la comprensión de las ideas sobre el pasado, el presente y, eventualmente escenarios futuros sugeridas por los jóvenes en sus narrativa "históricas" fue uno de los principales objetos de la investigación. La idea inicial fue la de llevar a cabo un estudio meramente exploratorio, cualitativo, para proporcionar datos empíricos a una propuesta de trabajo más elaborada, y por ello, eventualmente más consistente en sus conclusiones sobre la orientación temporal de los jóvenes estudiantes. Las propuestas de trabajo, lanzadas inicialmente a jóvenes de $10^{\circ}$ año y, a partir de 2004, a jóvenes de 9aano después de estudiar varios tópicos sobre el mundo contemporáneo, consistieron en escribir dos narrativas sobre la historia de los últimos cien años, la primera sobre Portugal y la segunda sobre el mundo. Los resultados abrieron tales posibilidades de análisis sobre el mundo conceptual de los jóvenes que las propuestas iniciales se mantuvieron en esencial para varias fases de la recolección de datos - tanto en escuelas de diversas regiones del país (para obtener alguna representatividad), como en otros países (para proporcionar algunos datos comparativos), y en fases más recientes (para, en un sentido de cierta forma longitudinal, proporcionar alguna comprensión de variación conceptual entre datos de 2004 a 2017). Actualmente, dentro de la misma propuesta metodológica, se investigan las narrativas históricas de alumnos de $9^{0}$ año en grupos multiculturales, formados sobretodo por jóvenes oriundos de familias inmigrantes de diversos países.

Un resultado que se ha relevado con acentuada regularidad en las anteriores fases de recolección en Portugal, es que las historias nacionales están más estructuradas y fundamentadas que las historias mundiales. De hecho, entre los jóvenes portugueses la Historia del país no sólo tiende a aparecer entretejida en una narrativa (versus lista cronológica o ideas fragmentadas) sino que también se centra en un segmento temporal que, al menos, va desde la dictadura (que ocurrió de 1930 a 1974) hasta la construcción de la democracia desde 1974 (con la revolución conocida entre los portugueses como "el 25 de Abril" o "la revolución de los claveles”) y que puede englobar también los tiempos actuales. Estas narrativas no son relatos meramente descriptivos, tienen algunos elementos explicativos (aunque convencionales), de carácter político, social y económico. Citando expresiones de alumnos, en tiempos de Salazar el pueblo

${ }^{25}$ Al Proyecto “Consciência Histórica - Teoria e Práticas (2003-2007)”, 06.11, 2013, URLhttp:// www.fct.pt/apoios/projectos/consulta/vglobal_projecto?idProjecto=49106\&sapiens=2002, siguió el proyecto "Consciência Histórica - Teoria e Práticas II (2017-2011), Consciência Histórica, 30.06.2018, URLwebs.ie.uminho.pt/conscienciahistorica/index56-2.html. 
vivía "momentos de tristeza, con la "voz" censurada e impedida", había "falta de libertad y de derechos, por ejemplo, las mujeres no podían votar", "Se vivía mal"; en 1974, "el pueblo "harto de la dictadura" se reveló", la "Revolución de los Claveles dio a Portugal de nuevo la libertad", en los años 1980 el país "entra en la Unión Europea” y "se desarrolla más”. Algunos textos son más abarcadores y narran la historia desde la implementación de la República en el inicio del siglo XX. Pero, en general, surgen también algunos textos que se consideran menos elaborados al asumir una forma narrativa más basada en comentarios generales o en el formato de lista cronológica, por lo tanto fragmentada y poco explicativa. El mensaje subyacente a los textos es en general optimista; sin embargo, en los datos recolectados en 2008 se percibe un tono de preocupación con menciones a la "crisis económica que el país vive en el presente", convicción que se va acentuando en 2011 y 2015 en un tono que invita a los políticos y a al pueblo "que están durmiendo pero tienen que levantarse". Las narrativas de 2017 nuevamente se expresan en términos optimistas y con un tono más sereno con relación al presente, muestran miradas más diversificadas sobre el país (una mirada crítica moderada que puede ser una señal de mayor madurez democrática).

Puede afirmarse que entre los jóvenes portugueses el mensaje nuclear nacional aunque no sea muy abierto al "otro" es al menos bastante diferente del que hubiera sido en tiempos de dictadura. Este, según lo juzgado por los textos escolares de pensamiento único, debería girar en torno a la victoria sobre los enemigos externos y de la defensa de un Portugal colonial perenne. El mensaje nuclear que atraviesa las narrativas de los jóvenes de nuestro tiempo es más abierto con relación al país: "la conquista de la libertad sobre la dictadura". Este elemento refuta la tesis de que los mensajes nucleares de las narrativas nacionales tienden a mantenerse entre generaciones y resisten los cambios de la historia vivida ${ }^{26}$. Sin embargo, es de destacar, que las cuestiones difíciles recientes como la guerra colonial y el proceso de descolonización en África, así como otras menos recientes como la esclavitud, raramente hacen parte de esas narrativas de los jóvenes portugueses. Tales cuestiones históricas van emergiendo paulatinamente, sobretodo en la última fase de recolección de datos, en 2017.

Con relación a la Historia del Mundo, en el plano substantivo las narrativas de los alumnos tienden a centrarse en el segmento temporal de la I y/o II guerras mundiales, seguido por acontecimientos mediáticos del siglo XXI.

${ }^{26}$ James Wertsch, Voices of Collective Remembering (Cambridge: CUP, 2002). 
Un considerable "agujero negro" entre los tiempos de las guerras mundiales y el presente - que, no obstante, corresponde a tópicos estudiados en el $9^{0}$ año- indica que asuntos como los "locos años de 1920", la crisis de 1929 y las diversas soluciones ensayadas para superarla (nacionalismos dictatoriales o frentes de izquierda), la "Guerra fría", entre otros, no fueron suficientemente significativos para esos jóvenes. Sin embargo, debe tenerse en cuenta que unos pocos alumnos dan cuenta de algunas de estas cuestiones en el plano mundial, como por ejemplo este joven que asistía a una escuela de Lisboa en 2008:
En 1914 ocurrió la 1ª guerra Mundial que duró hasta 1918. Esa guerra trajo inmensas consecuencias para todos los países que en ella intervinieron, es decir sociales, económicas y políticas. En la década del 30, en algunos países surgieron regímenes extremistas, como en Italia, Alemania, España y Portugal, liderados respectivamente por Mussolini, Hitler, Franco y Salazar. En el otro extremo de Europa (Rusia) apareció un régimen comunista, primero gobernado por Lenin y después por Stalin. Entre 1939 y 1945 se dio la $2^{\mathrm{a}}$ Guerra Mundial. Luego de esto se dio un proceso de reconstrucción de los países de Europa. En los últimos años se ha vivido un clima de terrorismo causado por el atentado hecho por Al-Queda en 2001 a las torres gemelas en Nueva York. Así, se han dado guerras en las que los UA aparecen involucrados, más específicamente en Afganistán y en Iraq (Alumno de $9^{\mathbf{o}}$ año de escolaridad, 15 años)

También se debe tener en cuenta que, en algunas narrativas de 2011 emerge a la vez, con alguna frecuencia, otro acontecimiento del siglo XX más allá de las guerras -la crisis de 1929- que indica la preocupación de entender la crisis económica que en ese momento se volvió más visible en Europa (y con repercusiones en el cotidiano de los propios jóvenes). La idea frecuente de que en el mundo exterior ("Yo nunca fui al mundo", así comenzaba la narrativa de un de los jóvenes) hay sobretodo "guerras, terrorismo y catástrofes, pero también ciencia y tecnología” parece ser persistente y no se presenta tan positiva en cuanto al mensaje nuclear nacional. Además de ser menos justificadas, en el plano estructural las narrativas mundiales se presentan frecuentemente de forma genérica, con comentarios sobre los males del mundo que señalan, además de las violencia, la desigualdad entre pueblos, la codicia y los problemas sociales y ambientales causados por ciertos grupos nacionales o sociales. En estos casos, el modo explicativo es frágil, ya que las narrativas carecen de fundamentación históricamente situada para las causas y consecuencias de los estados del mundo que presentan. Esto puede sugerir un sentido de identidad 
global mas vago del que de la identidad nacional, teniendo en cuenta que la Historia estudiada en los tres últimos años escolares de estos jóvenes (de $7^{\mathbf{0}}$ al $9^{\mathrm{a}}$ año) se centra en asuntos europeos y mundiales, en los cuales los temas nacionales surgen puntualmente integrados.

Estos estudios sobre narrativas de alumnos portugueses acerca de la Historia nacional y/o mundial son apenas un ejemplo, entre muchos otros, que se han desarrollado en las primeras décadas del siglo XXI orientados a la investigación en torno de la relación entre narrativa histórica y la formación de la conciencia histórica de los jóvenes ${ }^{27}$. Estudios en Brasil ${ }^{28}$, Reino Unido ${ }^{29} \mathrm{o}$ España $^{30}$-solo para hacer mención de algunos casos- han mostrado resultados que sugieren convergencias y especificidades entre si. Para citar sólo algunos puntos sobresalientes en las narraciones sobre la Historia contada por los jóvenes en cada país, los alumnos encuestados en Brasil y en España sugieren una clara identidad nacional ${ }^{31}$, a veces con criticas moderadas, en Inglaterra son más vagas las señales identitarias ${ }^{32}$. En los datos recolectados en Portugal y en Brasil en 2004-2005, en tareas idénticas los jóvenes portugueses y brasileros compartían una idea de progreso acerca de su país; no obstante, mientras que los jóvenes portugueses se mostraron como meros espectadores o "producto" de la historia hecha por otros, los jóvenes brasileños entraban en ella como agentes de cambio. Sería interesante volver al terreno hoy, al final de la segunda década de este siglo, e indagar en que medida tales perspectivas cambiaron o no.

${ }^{27}$ Debe tenerse en cuenta que el enfoque investigativo en las ideas de los alumnos ha ampliado los estudios para explorar las concepciones de varios agentes educativos, a saber, profesores y autores de textos escolares.

${ }^{28}$ Ver María Auxiliadora Schmidt, "Perspectivas da consciência histórica e da aprendizagem em narrativas de jovens brasileiros”. Revista Tempos Históricos, No. 12 (2008): 81-96.

${ }^{29}$ Ver Rosalyn Ashby et al., "British school history 'bigpictures' of the past” (Comunicação apresentada em 2008 AERA Annual Meeting, New York, March 2008).

${ }^{30}$ Ver Jorge Saíz y Ramón López Facal, “Competencias y narrativas históricas. El pensamiento histórico de estudiantes y futuros professores”. Revista de Estudios Sociales, No. 52 (2015): 87 101; Edda Sant, Neus González-Monfort, Antoni Santisteban Fernández, Joan Pagès Blanch y Montserrat Oller Freixa, "How do Catalan students narrate the history of Catalonia when they finish Primary Education", McGill Journal of Education, No 50, 2/3 (2015): 341-62.

${ }^{31}$ En el caso de España, si consideramos los resultados de los estudios señalados en la nota a pie 30, las señales de identidad nacional pueden orientarse hacia enfoques diferenciados.

${ }^{32}$ Para el caso de Irlanda del Norte, ver nota de pie de página 19. 


\section{Una nota final: de la investigación a la práctica del salón de clase}

Existen ya algunos resultados específicos sobre: a) cómo y hasta que punto los alumnos explican históricamente una situación dada; b) cómo analizan las fuentes y las cruzan para obtener inferencias sobre esa situación; c) cómo consideran, discuten y evalúan diferentes puntos de vista; d) cómo relacionan pasado y presente; e) cómo construyen hipótesis de futuro a la luz de esto. Todas estas cuestiones hacen parte de la labor investigativa en torno a la "narrativa histórica" que los sujetos producen y, por lo tanto, en torno de los modos y de las bases conceptuales que la Historia proporciona para decidir y actuar en el presente. Vale la pena estudiar las cuestiones de objetividad y subjetividad en Historia y el reconocimiento de los límites de la relatividad de las perspectivas, los niveles de comprensión del cambio y de la explicación histórica, ya que este interés promueve un posicionamiento más dialogante y humanista.

La investigación puede inspirarse en la batería de instrumentos ya utilizados en estudios anteriores o avanzar hacia nuevos abordajes metodológicos. El enfoque cualitativo con análisis inductivo de los datos, ha sido esencial para dar luz en profundidad sobre cómo los sujetos piensan en y sobre la Historia -aproximándose de cierto modo al oficio de los historiadores al interpretar sus fuentes-. En este paradigma de estudios exploratorios y descriptivos, el análisis cuantitativo adicional ha contribuido para identificar tendencias en los resultados y sugerir nuevos desafíos. Además, el análisis en profundidad del pensamiento histórico, desde la óptica de esta investigación en educación histórica, exige disponibilidad de tiempo y una orientación epistemológica clara, razón por la cual las muestras participantes han de ser reducidas. En una época de contabilización de los resultados, será necesario procurar conciliar mejor los paradigmas cualitativo y cuantitativo en este tipo de investigación, con el fin de permitir una diseminación aún más amplia de lo que significa desarrollar el pensamiento histórico de los jóvenes.

Por ahora, los resultados de los estudios aquí presentados de forma muy resumida, revelan que los alumnos pueden pensar de forma autónoma acerca del pasado, incluso si son educados históricamente solo en el nivel de comprensión de contenidos substantivos. Aunque la Historia sea frecuentemente enseñada según un modelo epistemológico de verdad factual única y casi fija, los alumnos toman lo que les es más significativo y, algunas veces, se muestran capaces de argumentar a favor o en contra de algunas versiones de la Historia, con uso de criterios de segundo orden relativamente validos. En efecto, aunque muchos presentan argumentos poco elaborados, a veces cercanos de 
posturas positivistas de verdad consensual única y otras veces vinculados a un relativismo basado en un punto de vista acrítico, otros alumnos han mostrado un pensamiento a un nivel más sofisticado, integrando de alguna forma la consideración de diversidad de perspectivas que deben ser confirmadas o refutadas a la luz de la evidencia.

Estas conclusiones provienen de investigaciones que incluyen varios estudios sobre ideas de los jóvenes en la Historia, algunas de ellas anteriores a las que se centran en las narrativas como modo predominante de producción historiográfica. Es de resaltar que las tareas colocadas a los jóvenes en ambientes de investigación podrán servir de inspiración para las tareas de los alumnos en las clases de Historia. Para realizar una posible aplicación de tareas a niños y/o jóvenes en las clases de Historia, se propone un modelo de clase-taller de Historia de inspiración constructivista que involucra ${ }^{33}$ :

- Propuestas de cuestiones orientadoras, problematizadoras, sobre un tema histórico;

- Exploración de ideas previas de los alumnos acerca del tema a estudiar;

- Consideración de los niveles de ideas previas reveladas por los alumnos para estimular su profundización o la reformulación conceptual de las mismas a lo largo de la clase;

- Momentos de trabajo de los alumnos -individual o en pequeños grupos (de dos a cinco), en discusión plenaria, etc.- con asignación de tareas, siempre que sea posible basadas en la interpretación de fuentes de la Historia;

- Acompañamiento (monitorización) del trabajo de los alumnos por parte del profesor;

- Momentos de meta-cognición, en los que los alumnos reflexionan y ganan conciencia sobre lo que ellos propios aprendieron y sobre lo que les gustaría aprender más (o no) sobre el tema.

En esta primera mitad del siglo XXI, será pues necesario estimular el pensamiento histórico de los jóvenes a partir de lo que ya se conoce acerca de qué y cómo piensan hoy. Para ayudarlos a pasar de lo familiar a lo extraño, será necesario conocer sus ideas más allá de la regurgitación de contenidos fijos. En clase, en varios sentidos es un ejercicio saludable colocar (a veces) a los alumnos

${ }^{33}$ Isabel Barca, "A aula oficina em História: do projecto à avaliação", en Para uma Educação Histórica de qualidade. Actas das IV Jornadas Internacionais de Educação Histórica, coord. Isabel Barca (Braga: Centro de Investigação em Educação, Universidade do Minho, 2004), 131-144. 
en situación de escribir "por su propia cabeza”, su versión de la Historia con la garantía de que no van a ser clasificados por criterios de correcto-incorrectoincompleto en la tarea, ya que: 1) da la oportunidad a los alumnos de clarificar y expresar su pensamiento histórico; 2) sirve de diagnóstico para que el profesor oriente su trabajo pedagógico en una perspectiva constructiva, al percibir un poco mejor el mundo conceptual de quien aprende; 3) contribuye al autoestima de los alumnos (porque sus ideas cuentan y tienen valor); 4) crea un ambiente de integración saludable en la clase. Esta propuesta de investigación incluye un esfuerzo conceptual, por parte del formador, de buscar entender las ideas de los jóvenes desde la perspectiva de conocer y monitorizar mejor la base histórica que ya dominan o no. El modelo de Clase-taller no pretende ser una biblia, por el contrario debe ser entendido en el marco de flexibidad curricular. Algunos docentes ya colocaron en práctica esta propuesta, con resultados satisfactorios. No obstante, se necesita más investigación sobre los sentidos que los profesores de Historia , en general, atribuyen a estas repercusiones de la investigación en la enseñanza y en la evaluación. En algunos países, los textos escolares ya ofrecen nuevas propuestas de exploración de las ideas previas de los alumnos, interpretación cruzada de fuentes y preguntas problematizadoras algunas veces inspiradas directamente en estudios sistemáticos en educación histórica. Desde una perspectiva de optimismo moderado, se considera que la semilla esta lanzada: oír diferentes voces -incluyendo las voces de los alumnos y de profesores- en la búsqueda de respuestas consistentes sobre la vida de los seres humanos en varios tiempos es un desafío esencial para la Educación de los jóvenes en una sociedad plural.

\section{Referencias bibliográficas}

\section{Fuentes secundarias}

Abrantes, Paulo. Currículo nacional do Ensino Básico - Competências essenciais. Lisboa: DEB, Ministério da Educação, 2001.

Alves, Olinda, "Concepções de professores e de alunos sobre significância histórica - um estudo no $3^{\mathbf{0}}$ ciclo do ensino básico”. Tese de Maestría, Universidade do Minho, 2007.

Ashby, Rosalyn y Lee, Peter, "Children's concepts of empathy and understanding in history”. En The History Curriculum for Teachers. Editado por Christopher Portal, 62-88. London: The Falmer Press, 1987.

Ashby, Rosalyn, "O conceito de evidência histórica: exigências curriculares e concepções dos alunos”. En Educação Histórica e Museus. Actas das II Jornadas 
Internacionais de Educação Histórica, 37-57. Coordenado por Isabel Barca. Braga: CIEd, Universidade do Minho, 2003.

Ashby, Rosalyn, Stuart Foster, Jono Howson y Peter Lee, "British school history 'big pictures' of the past”. Apresentação ao 2008 AERA Annual Meeting, New York, March 2008.

Atkinson, R.. Knowledge and explanation in history: An introduction to the philosophy of history. London: Macmillan, 1978.

Barca, Isabel. O Pensamento histórico dos jovens. Braga: CEEP, Universidade do Minho, 2000.

Barca, Isabel, "A aula oficina em História: do projecto à avaliação”. En Para uma Educação Histórica de qualidade. Actas das IV Jornadas Internacionais de Educação Histórica, 131-144. Coordenado por Isabel Barca. Braga: Centro de Investigação em Educação, Universidade do Minho, 2004.

Barton, Keith, "Ideias das crianças acerca da mudança através dos tempos: resultados de investigação nos Estados Unidos e na Irlanda do Norte”. En Perspectivas em Educação Histórica. Actas das I Jornadas Internacionais de Educação Histórica”, 55-68. Coordenado por Isabel Barca. Braga: CEEP, Universidade do Minho, 2001.

Barton, Keith y McCully, Alan. "When History teaching really matters - Understanding the impact of school intervention on students' neighbourhood learning in Northern Ireland”. International Journal of Historical Learning Teaching and Research, 8, n. 1 (2009): 28-46.

Castro, Júlia, "A Interculturalidade e o Pensamento Histórico dos Jovens". Tesis de doctorado, Universidade do Minho, 2006.

Carvalho, Manuela y Barca, Isabel. "Gli studenti e le fonti in tre paesi europei". I Quadreni di Clio'92, 13 (2014): 95-114.

McCully, Alan. "A question of identity? Purpose, policy and practice in the teaching of history in Northern Ireland and the Republic of Ireland". International Journal of Historical Learning Teaching and Research, 11, n. 2 (2013): 145-158.

Cercadillo, Lis, “Significance in history: students' ideas in England and Spain”. En Raising Standards in History Education. International Review of History Education, 116-145. Editado por Alaric Dickinson, Peter Gordon y Peter Lee. London: Woburn Press, 2001.

Chaves, Fátima, "A significância de personagens históricas na perspectiva de alunos portugueses e brasileiros”. Tesis de Maestría, Universidade do Minho, 2006.

Collingwood, R. G. The Idea of History. Oxford: Clarendon Press, 1946.

Dickinson, Alaric y Lee, Peter. History teaching and historical understanding. London: Heineman, 1978.

Dray, William. Perspectives on history. London: Routledge and Kegan Paul, 1980.

Fronza, Marcelo, "Os jovens e as ideias de objetividade e verdade na aprendizagem histórica sobre o passado a partir das histórias em quadrinhos”. En Educação e Consciência Histórica na Era da Globalização, 179-200. Coordinado por Isabel Barca. Braga: CIED, Universidade do Minho/Associação de Professores de História, 2011. Fulbrook, Mary. Historical theory. London: Routledge, 2002. 
Gago, Marília, "Children's understanding of historical narrative in Portugal". En Understanding History: Recent research in in history education, 83-97.Editado por Rosalyn Ashby, Peter Gordon y Perter Lee. London: Routledge Falmer 2005.

Lagarto, Mariana, "Desenvolver e avaliar competências em História. Um estudo com professores do $3^{0}$ ciclo do ensino básico”. Tesis de doctorado, Universidade do Minho, 2017.

Lee, Peter. “Por que aprender História?'. Educar em Revista, 42 (2011): 19-42.

Lloyd, Christopher. The structures of history. Oxford: Basil Blackwell,1993.

Machado, Elvira. "Mudança em História - concepções de alunos do 7. ano de escolaridade”. Tesis de Maestría, Universidade do Minho, 2006.

Martin, Raymond. The past within us. Princeton: Princeton University Press, 1980.

Monsanto, Márcia, "Concepções de alunos sobre Significância Histórica no contexto da História de Portugal: um estudo com alunos do $3^{\circ}$ ciclo do Ensino Básico”. Tesis de Maestría, Universidade do Minho, 2004.

“Projeto Consciência Histórica - Teoria e Práticas (2003-2007)”, URL http:// www.fct.pt/apoios/projectos/consulta/vglobal_projecto?idProjecto=49106\&sapiens $=2002$.

“Projeto Consciência Histórica - Teoria e Práticas II (2017-2011)”. Consciência Histórica, URL webs.ie.uminho.pt/conscienciahistorica//index56-2.html.

Rüsen, Jörn, “The development of narrative competence in historical learning: an ontogenetic hypothesis concerning moral consciousness”. En Studies in metahistory. Editado por Peter Duvenage, 63-84. Pretoria: Human Sciences Research Council, 1993.

Rüsen, Jörn. A Razão da História. Teoria da História: os fundamentos da ciência histórica. Brasília: UniB, 2001.

Rüsen, Jörn. “Humanismo Intercultural: ideia e realidade”. En Humanismo e Didática da História, 133-152. Editado por Maria Auxiliadora Schmidt, Isabel Barca, Marcelo Fronza y Lucas Pydd Nechi. Curitiba: W. A. Editores, 2015.

Saíz, Jorge y López Facal, Ramón. “Competencias y narrativas históricas. El pensamiento histórico de estudiantes y futuros professores". Revista de Estudios Sociales, 52 (2015): 87-101.

Sant, Edda, Neus González-Monfort, Antoni Santisteban Fernández, Joan Pagès Blanch, y Montserrat Oller Freixa. "How do Catalan students narrate the history of Catalonia when they finish Primary Education”, McGill Journal of Education, 50, n. 2/3 (2015): 341-62.

Schmidt, M. Auxiliadora. "Perspectivas da consciência histórica e da aprendizagem em narrativas de jovens brasileiros”. Revista Tempos Históricos, 12 (2008): 81-96.

Schmidt, Maria Auxiliadora, Isabel Barca, Carla Gomes da Silva y Vaneska Pegoraro. Aprendizagem Histórica: Catálogo Seletivo de Teses e Dissertações Brasileiras e Portuguesas. Curitiba: W.A. Editores, 2018.

Seixas, Peter. "Mapping the terrain of Historical Significance”. En Theory and Research in Social Education, 61, n. 1 (1997): 22-27. 
Shemilt, Denis. History 13-16: Evaluation Study. Edinburgh: Holmes McDougall, 1980.

Shemilt, Denis. "Beauty and the philosopher: Empathy in history and the classroom". En Learning history, 39-84. Editado por Alaric Dickinson, Peter Lee y Peter Rogers. London: Heinemann, 1984.

Shemilt, Denis. "Adolescent ideas about evidence and methodology in history". En The History Curriculum for Teachers, 39-61. Editado por Christopher Portal. London: The Falmer Press, 1987.

Souza, Eder, "Cinema e Educação histórica: Jovens e a sua relação com a História nos filmes". Tesis de Doctorado, Universidade Federal do Paraná, 2014.

Veríssimo, Helena, "A avaliação de competências históricas através da interpretação da evidência". Tesis de doctorado, Universidade do Minho, 2013.

Wertsch, James. Voices of Collective Remembering. Cambridge: CUP, 2002. 